\title{
Diagnóstico da arborização após a ocorrência de evento climático extremo
}

As regiões Sul e Sudeste do país são as que vêm sendo mais afetadas pela ocorrência de eventos climáticos extremos, como tempestades e vendavais. Tais ocorrências provocam prejuízos severos, incluindo queda de árvores. O objetivo desta pesquisa foi realizar o diagnóstico da arborização danificada no campus da Universidade Federal de Viçosa após ocorrência de tempestade. A coleta de dados ocorreu em outubro de 2019, imediatamente após a ocorrência do evento climático. Foi realizada contagem, identificação botânica e avaliação do estado fitossanitário dos indivíduos danificados, bem como a determinação do grau de dano sofrido (leve, moderado, severo e irreversível), o local de ruptura e a medição de parâmetros dendrométricos daqueles indivíduos mais danificados. No total, 145 indivíduos que compõe a arborização apresentaram algum dano ocasionado pela tempestade, de 29 espécies, o que corresponde a $13 \%$ do total de indivíduos existentes no campus. A família com maior ocorrência de danos foi Bignoniaceae e as espécies mais afetadas foram Spathodea campanulata (29\%), Tibouchina granulosa (22\%) e Licania tomentosa (15\%). A maioria apresentou ruptura nos galhos $(61 \%), 23 \%$ na base ou tronco e $4 \%$ no ápice, compondo o total de $36 \%$ de ocorrências com dano irreversível. Cerca de $30 \%$ dos indivíduos apresentavam problemas fitossanitários e $70 \%$ corresponde a plantios em áreas de calçadas, o que permite associar o maior risco de queda às árvores em locais com menor área de canteiro, espaço disponível para o desenvolvimento das raízes e área disponível para crescimento.

Palavras-chave: Risco de Queda; Ventos; Potencial de Falha.

\section{Afforestation diagnosis after extreme weather event}

The South and Southeast regions of the country are the ones that have been most affected by extreme weather events such as thunderstorms and windstorms. Such occurrences cause severe damage, including tree fall. The objective of this research was to diagnose damaged afforestation on the campus of the Federal University of Viçosa after a storm. Data collection took place in October 2019, immediately after the occurrence of the weather event. Counting, botanica identification and evaluation of the phytosanitary status of the damaged individuals were performed, as well as the degree of damage suffered (mild, moderate, severe and irreversible), the rupture site and the dendrometric parameters of those most damaged individuals. A total of 145 individuals that make up the afforestation had some storm damage of 29 species, which corresponds to $13 \%$ of the total on campus. The family with the highest occurrence of damage was Bignoniaceae and the most affected species were Spathodea campanulata (29\%), Tibouchina granulosa (22\%) and Licania tomentosa (15\%). Most presented rupture in the branches $(61 \%), 23 \%$ in the base or trunk and $4 \%$ in the apex, making a total of $36 \%$ of occurrences with irreversible damage. About $30 \%$ of the individuals had phytosanitary problems and $70 \%$ correspond to plantations in sidewalk areas, which allows to associate the higher risk of falling to trees in places with smaller area of soil, available space for root development and area available for growth.

Keywords: Fall Risk; Winds; Failure Potential.

Topic: Planejamento, Gestão e Políticas Públicas Ambientais

Reviewed anonymously in the process of blind peer.
Received: $10 / 12 / 2019$

Approved: $26 / 02 / 2020$

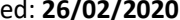

Tamilis das Graças Emerick

Universidade Federal de Viçosa, Brasil http://lattes.cnpq.br/8003132544772365

http://orcid.org/0000-0002-4158-0369

etamilis13@gmail.com

Angeline Martini

Universidade Federal de Viçosa, Brasil

http://lattes.cnpq.br/1793083628826054

http://orcid.org/0000-0002-4500-1221

martini.angeline@gmail.com 


\section{INTRODUÇÃO}

O reconhecimento e avaliação regular dos riscos naturais que interferem na segurança da população são passos indispensáveis para o sucesso do planejamento e ordenamento do território (RIBEIRO et al., 2011). Nesse sentido, a arborização urbana se configura como elemento primordial no planejamento e monitoramento das zonas urbanas, uma vez que gera inúmeros benefícios, sendo indispensável nesse ambiente, ao mesmo tempo em que constitui risco de alta prioridade.

Apesar da importância da arborização para a qualidade de vida, o planejamento e a gestão quando realizados de forma inadequada podem promover riscos para a população e resultar em perdas materiais ou mesmo fatalidades (BRESSANE et al., 2018). O aumento da ocorrencias de quedas de árvores nos centros urbanos tem se intensificado nos últimos anos, causada por diversos fatores, como: chuvas fortes e longas, podas irregulares, rajadas de vento e raiz instável (SAMPAIO et al., 2010). Tem-se ainda outros fatores antrópicos que podem interferir diretamente nas chances de quedas de árvores, como: o corte de raízes, uso indevido de espécies e a compactação do solo (MOSER et al., 2010). A queda de árvores e/ou galhos é um problema atual e muito grave em várias cidades do mundo (PEREIRA, 2011).

No Brasil não é diferente, sendo cada vez mais comuns relatos de casos onde a queda de árvores gera perdas humanas ou materiais, geralmente após eventos de chuvas ou vendavais. Só na capital mineira, Belo Horizonte, em 2018, foram suprimidas mais de 10 mil árvores em risco de queda, sendo investidos cerca de 15 milhões de reais para podas e remoções, além de registrada uma morte provocada pela queda de árvore durante tempestade (BELO HORIZONTE, 2018). Já na capital de São Paulo, foram 2,5 mil árvores perdidas em tempestades de verão, no ano de 2019, tendo a prefeitura gasto cerca de 200 mil reais com indenizações a proprietários de veículos atingidos (SÃO PAULO, 2019).

Segundo Sampaio et al. (2010), as regiões Sul e Sudeste do país são as que vêm sendo mais afetadas pela ocorrência de eventos climáticos extremos, em especial durante o verão, como tempestades e vendavais. Estes podem influenciar na estabilidade das árvores, causando queda de galhos ou da árvore toda, com consequente dano físico ao espaço urbano.

James (2003) ressalta que o vento é o maior gerador de tensão a que as árvores têm de resistir. Há ainda outros agravantes, visto que em ambiente urbano as circunstâncias em que as árvores se desenvolvem são muito diferentes das encontradas nos ambientes naturais ou zonas rurais. A luminosidade, características do solo, qualidade do ar e o clima são menos propícios para a fisiologia das árvores, condições que aliadas aos efeitos da ilha de calor urbano com baixas condições de insolação e temperaturas mais altas, podem reduzir o ciclo de vida dos indivíduos arbóreos (LOPES et al., 2008).

Para as árvores situadas em zonas de calçamento, as condições são ainda mais limitantes comparativamente com aquelas plantadas em áreas com grande espaço permeável disponível, uma vez que estão expostas a situações de maior restrição para o crescimento vegetativo (FABIÃO, 1996, citado por NILSSON et. al., 2000).

Por isso, a fim de minimizar os riscos, se faz importante a criação de um sistema de gestão específico 
para o estrato arbóreo das áreas urbanas, além do incremento de uma política de risco com portarias que tratem de pontos como: espécies a serem implantadas, local de implantação, limitações de infraestrutura, manejo adequado e monitoramento (DUNTEMANN, 2006). O autor considera, ainda, a elaboração de um mapa de risco como outro fator importante, que permite o monitoramento das espécies com algum potencial de dano e agilidade para ações em eventos de tempestade. Neste contexto, para dar suporte à elaboração das medidas necessárias que visam suavizar tal problema, este estudo objetivou realizar o diagnóstico da arborização danificada após evento climático severo de chuvas e ventos ocorrido em outubro de 2019 no campus da Universidade Federal de Viçosa, MG.

\section{METODOLOGIA}

O estudo foi conduzido no campus da Universidade Federal de Viçosa, na cidade de Viçosa, em Minas Gerais, situado nas coordenadas $20^{\circ} 45^{\prime} 37^{\prime \prime} \mathrm{S} 42^{\circ} 52^{\prime} 04^{\prime \prime} \mathrm{O}$, totalizando uma área de 1.359 ha (PORTES et al., 2009). O município de Viçosa está inserido na região da Zona da Mata Mineira, é caracterizado por temperatura média anual de $21,8^{\circ} \mathrm{C}$ (CASTRO et al., 1973) e precipitação média anual de cerca de $1200 \mathrm{~mm}$ (SOARES JÚNIOR, 2000), o clima é do tipo Cwa, segundo a classificação de Koppen, e, portanto, mesotérmico com verões quentes e chuvosos e invernos frios e secos. O bioma predominante é Mata Atlântica, composto por Floresta Estacional Semidecidual (VELOSO et al., 1991).

O campus de Viçosa é considerado um dos mais bonitos do Brasil, principalmente por conta da ornamentação vegetal, a qual é composta em sua maioria pela arborização de vias e calçadas. Possui uma ampla estrutura acadêmica, contanto com 11.367 alunos matriculados, além de funcionários e população em geral que utilizam o espaço para diversos fins, como de lazer e esporte.

Na noite do dia 25 de Outubro de 2019 o município sofreu com um evento climático extremo, com fortes chuvas e rajadas de vento. As $21 \mathrm{~h}$ foram registradas pelo INMET rajadas de vento com velocidade de $82,4 \mathrm{~km} / \mathrm{h}$ e uma precipitação acumulada de $67,0 \mathrm{~mm}$, para essa hora. Ao todo, em menos de cinco horas choveu $112,4 \mathrm{~mm}$, mais do que todo o esperado para o mês e a velocidade do vento excedeu a média anual local, que é de $14,34 \mathrm{~km} / \mathrm{h}$. No município foram observados diversos danos desencadeados pelo evento climático, como queda e dano de árvores, destelhamento de casas, falta no abastecimento de energia e comunicação, além de queda de placas e postes.

No dia seguinte à tempestade foi realizada a coleta de dados na área de estudo. Fizeram parte do levantamento apenas árvores, arbustos e palmeiras que compõem o paisagismo do campus, ou seja, excluíram-se as ocorrências existentes nos fragmentos florestais naturais e margem da lagoa. As variáveis analisadas e registradas em ficha de campo foram: a) Quantificação dos indivíduos danificados; b) Identificação botânica das espécies; c) Localização da ocorrência - determinação do tipo de local ocupado calçada, estacionamento, conexões entre edificações, áreas de lazer e ornamentação, etc.; d) Identificação do ponto de ruptura - raiz, base, tronco, ápice do tronco e galhos; e) Determinação do grau de dano - leve, ruptura de galhos finos (menores que $20 \mathrm{~cm}$ de diâmetro); moderado, ruptura de até 2 galhos maiores que $20 \mathrm{~cm}$ de diâmetro; severo, ruptura de 3 ou mais galhos maiores que $20 \mathrm{~cm}$ de diâmetro; irreversível, queda 
de toda a árvore ou perda da porção apical; f) Avaliação do estado fitossanitário - análise visual que consistiu na identificação de problemas existentes, como presença de cavidades, podridão do lenho, corpos frutíferos de fungos, sinais de atividades de cupins, presença de insetos e etc..

Os indivíduos que sofreram danos irreversíveis tiveram sua direção de queda definida e mensuração de alguns parâmetros dendrométricos. A determinação da direção de queda foi realizada com auxílio de uma bússola, quanto aos parâmetros dendrométricos foram medidos com trena o diâmetro da base do tronco e a altura.

\section{RESULTADOS}

No total foram encontrados 145 indivíduos arbóreos com algum grau de dano ocasionado pela tempestade distribuídos em 29 espécies de 19 famílias, o que corresponde a 13\% do total da arborização urbana do campus em número de indivíduos. Destes indivíduos $96 \%$ são árvores, 0,7\% arbustos e 3,3\% palmeiras.

A família com maior número de ocorrências foi Bignoniaceae e as espécies mais afetadas foram, respectivamente, Spathodea campanulata (29\%), Tibouchina granulosa (22\%), Licania tomentosa (15\%) e Handroanthus serratifolius (6\%) (Figura 1). A família Bignoniaceae, mais representativa no estudo, contribuiu com 36\% no total de indivíduos danificados, seguida por Melastomataceae com 22,5\%.

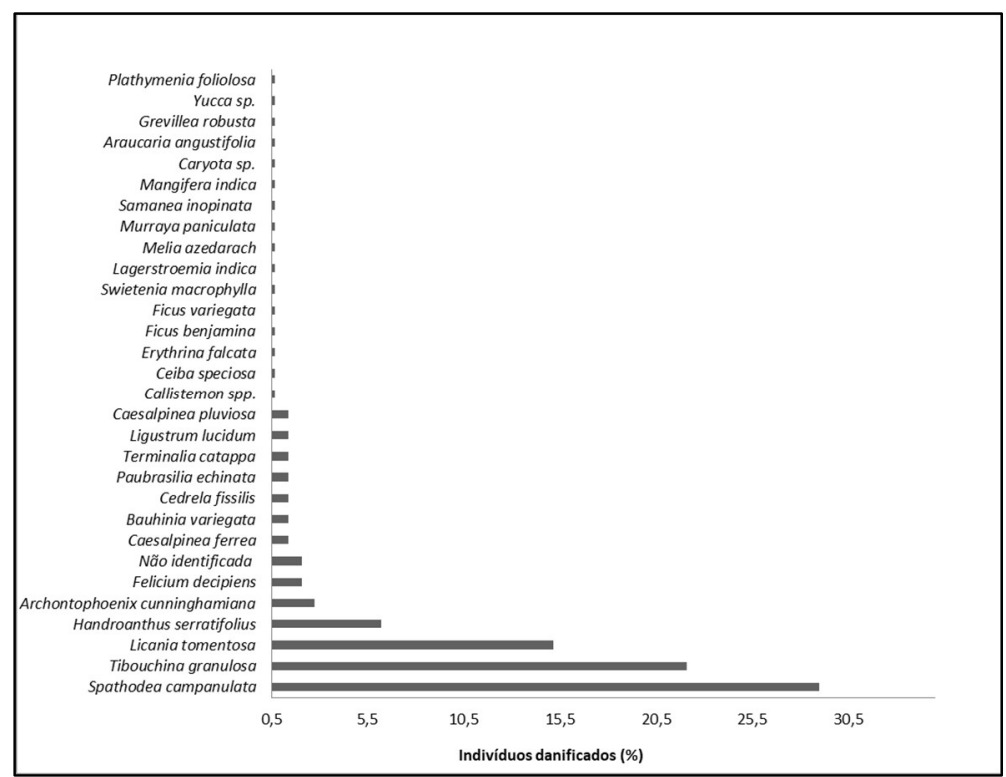

Figura 1: Espécies danificadas no campus da Universidade Federal de Viçosa na tempestade de 25 de Outubro de 2019, em Viçosa/MG.

Não é comum haver relatos de queda de palmeiras em ambientes urbanos após eventos meteorológicos, no entanto, foi registrada a queda de cinco exemplares de Archontophoenix cunninghamiana no presente estudo. Apresentaram grau de dano considerado como irreversível $36 \%$ dos indivíduos (Tabela 1), 31\% apresentaram grau moderado, 20\% leve e 13\% severo.

Os indivíduos mais severamente afetados foram na maioria de pequeno porte (42\%), enquanto que $19 \%$ eram de médio porte e $61 \%$ considerados como de grande porte. A maior parte apresentou altura entre 
3,0 e 18,0 m e diâmetro basal entre 0,13 e 1,4 m. Do total de indivíduos afetados de forma irreversível, 50\% pertencem à flora nativa do Brasil, enquanto $50 \%$ correspondem a indivíduos exóticos.

Tabela 1: Espécies arbóreas do campus UFV avaliadas com grau de dano irreversível. DB = Diâmetro na base do tronco médio $(\mathrm{m}) ; \mathrm{H}=$ Altura total média $(\mathrm{m}) ; \mathrm{N}=$ Número de indivíduos afetados.

\begin{tabular}{|l|l|l|l|l|l|}
\hline Espécie & Nome Popular & Família & DB & H & N \\
\hline Araucaria angustifolia & Pinheiro-do-Paraná & Araucariaceae & 0,29 & 8,8 & 1 \\
\hline Archontophoenix cunninghamiana & Palmeira-australiana & Arecaceae & 0,25 & 18,0 & 4 \\
\hline Bauhinia variegata & Pata-de-vaca & Fabaceae & 0,24 & 11,0 & 1 \\
\hline Caesalpinea ferrea & Pau-ferro & Fabaceae & 0,48 & 10,5 & 1 \\
\hline Caryota sp. & Palmeira-rabo-de-peixe & Arecaceae & 0,13 & 5,0 & 1 \\
\hline Clitoria fairchildiana & Sombreiro & Fabaceae & 0,36 & 14,0 & 1 \\
\hline Filicium decipiens & Felício & Sapindaceae & 0,18 & 5,0 & 1 \\
\hline Handroanthus serratifolius & Ipê-amarelo & Bignoniaceae & 0,52 & 12,5 & 2 \\
\hline Lagerstroemia indica & Resedá & Lythraceae & 0,30 & 7,0 & 1 \\
\hline Licania tomentosa & Oiti & Chrysobalanaceae & 0,38 & 12,0 & 4 \\
\hline Mangifera indica & Mangueira & Anacardiaceae & 0,42 & 9,0 & 1 \\
\hline Murraya paniculata & Murta & Rutaceae & 0,10 & 3,0 & 1 \\
\hline Spathodea campanulata & Bspatódea & Bignoniaceae & 1,20 & 13,5 & 16 \\
\hline Swietenia macrophylla & Mogno-africano & 0,44 & 12,0 & 1 \\
\hline Terminalia catappa & Sete-copas & Combretaceae & 0,48 & 15,0 & 1 \\
\hline Tibouchina granulosa & Quaresmeira & Melastomataceae & 0,37 & 6,2 & 13 \\
\hline
\end{tabular}

As direções de queda predominantes entre os indivíduos com dano irreversível foram: rnor-noroeste $(32,5 \%)$, seguida por noroeste $(25,0 \%)$, nordeste $(22,5 \%)$, oés-noroeste $(12,5 \%)$ e lés-nordeste $(7,5 \%)$. A rajada de vento mais forte, registrada pela estação meteorológica, no momento da tempestade originou-se da direção sul-sudeste, seguida por sul-sudoeste e oes-sudoeste, respectivamente, evidenciando a correlação entre o sentido de queda das árvores e a direção do vento.

Com relação à localização do dano, 61\% dos indivíduos apresentaram ruptura de galhos, $23 \%$ tiveram ponto de ruptura no tronco, $12 \%$ na base. Destes, a maioria (70\%) apresentou descolamento entre raiz e tronco e uma menor parte (30\%) teve o sistema radicular removido do solo. Cerca de $30 \%$ das árvores apresentaram, ainda, algum problema fitossanitário agravante, como cavidades, apodrecimento e galerias de insetos broqueadores. Desse percentual, $81 \%$ são de indivíduos da espécie Spathodea campanulata. A maioria dos indivíduos relatados com danos irreversíveis (70\%) estava localizada em calçadas, enquanto apenas $17 \%$ estavam em áreas de estacionamento e 13\% em jardins e demais locais urbanos.

\section{DISCUSSÃO}

As famílias mais abundantes no estudo compreendem diversas espécies amplamente utilizadas para fins de arborização urbana no Brasil, segundo Lorenzi (2002), reforçando a preocupação com a composição das florestas urbanas na atualidade e a necessidade de redefinir seus padrões de planejamento.

Espécies como Spathodea campanulata e Tibouchina granulosa, compreendidas por essas famílias, contribuem com mais de $50 \%$ dos indivíduos danificados, sendo provável que possuam lenho de baixa resistência mecânica e devam ser evitadas em ambientes urbanos, pelo risco de queda que apresentam diante de eventos extremos.

Vale (2005) confirma essa hipótese, ao estudar a densidade de resíduos da arborização urbana, onde observou para a Espatódea (Spathodea campanulata) baixos valores de densidade básica, indicando madeira 
mole, leve e de baixa resistência. Para Tibouchina granulosa Palharini et al. (2018) encontrou valor de densidade básica igual a $0,48 \mathrm{~g} / \mathrm{cm}^{3}$, o que é considerado por Silveira et al. (2013) madeira de baixa densidade (abaixo de $0,55 \mathrm{~g} / \mathrm{cm}^{3}$ ). É comum, ainda, que indivíduos de maior porte constituam a classe mais representativa em eventos de queda, no entanto informações sobre o tamanho dos indivíduos em meio urbano são raras.

$\mathrm{Na}$ cidade de Manchester, por exemplo, foi observado que "os danos às estruturas urbanas aumentavam de acordo com o tamanho das árvores" (WONG et al., 1988, citado por KLECHOWICZ, 2001). Isso sustenta o princípio de que árvores grandes não devem ser confinadas em espaços pequenos e pode constituir uma importante ferramenta na avaliação de risco de queda.

Quanto à origem dos indivíduos danificados no evento, o resultado apresentado refuta a ideia de que espécies nativas são mais eficientes para o fim de arborização de ruas, uma vez que apresentam mesmo grau de susceptibilidade à queda diante do evento climático em estudo, reforçando a proposta de que espécies exóticas bem adaptadas às condições edafoclimáticas locais e de aspectos silviculturais bem conhecidos podem contribuir para o sucesso da arborização urbana.

A ocorrência de um maior número de queda de árvores nativas na arborização já foi observada em outros estudos como o de Klechowicz (2001), em Curitiba/PR, onde metade das espécies com maior frequência de queda também se tratavam de nativas brasileiras. Em Belo Horizonte, Souza et al. (2019) relataram valor próximo a $60 \%$ nos registros de queda por espécies nativas.

"A maioria das árvores começa a cair com ventos de 25 até $54 \mathrm{~km} / \mathrm{h}$ de velocidade" (OLIVEIRA et al., 2007), valor muito abaixo do máximo registrado no momento do evento climático em estudo, onde é comum a queda de galhos, sendo a bifurcação o principal ponto de rompimento dessa fração, estando ainda associada à arquitetura da espécie e a forma de inserção do galho no tronco principal, uma vez que galhos inseridos de forma perpendicular podem apresentar maior resistência do que aqueles inseridos de forma oblíqua (KLECHOWICZ, 2001). Para a mesma autora, muitos acidentes com queda de galhos ou mesmo troncos podem estar relacionados ao apodrecimento interno, muitas vezes ocasionado por podas excessivas.

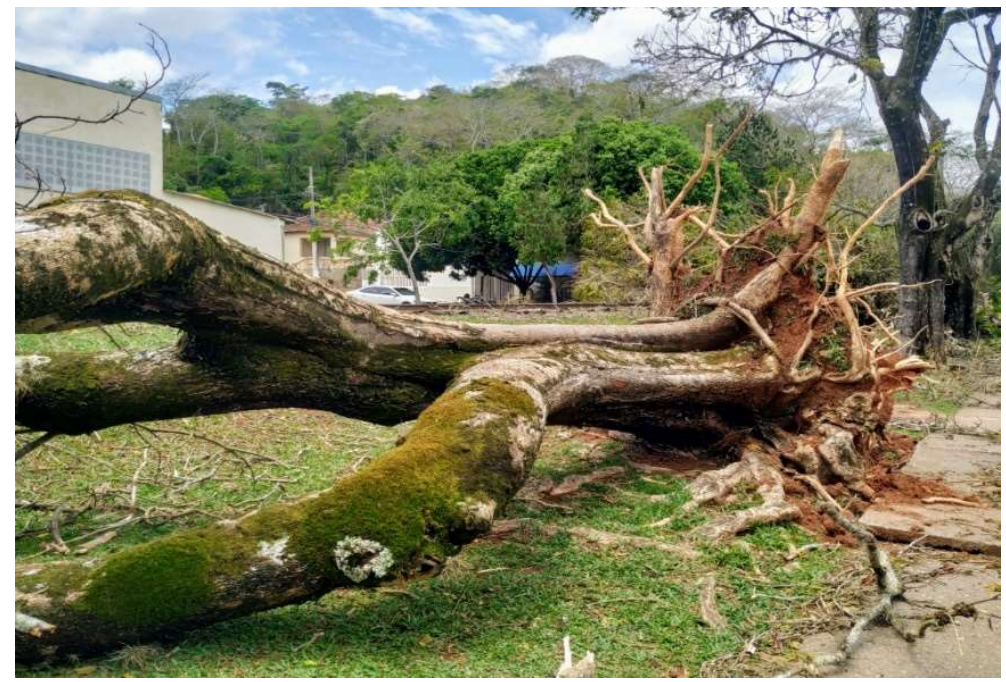

Figura 2: Indivíduo de Spathodea campanulata arrancado com o sistema radicular em tempestade ocorrida na Universidade Federal de Viçosa, em 25 de Outubro de 2019. 
Deve-se ainda chamar a atenção para a presença de indivíduos com ponto de ruptura na porção basal, tido como o padrão mais comum de falha estrutural em espécies arbóreas, segundo Edberg et al. (1999). Nos estudos de Souza et al. (2019) em Belo Horizonte - MG e de Klechowicz (2001) em Curitiba - PR esse comportamento também esteve presente na maioria dos casos de queda. Isso constitui um grande problema, visto que em métodos de análise visual de risco de queda, o sistema radicular é o ponto mais frágil da avaliação e, comumente, não é visível para tal (BRAZOLIN, 2009), o que impede ações antecipadas de mitigação do risco. Quanto à avaliação da condição fitossanitária dos indivíduos afetados, a idade avançada dos exemplares de Spathodea campanulata somada ao manejo inadequado pode explicar a alta incidência destes nessa condição.

Oliveira et al. (2007) relatam que, na década de 90, em Lisboa, Portugal, a queda de árvores era consequência de idade avançada e do aumento da poluição, que influenciavam nas condições fitossanitárias das plantas. Young (1984) acredita que o risco de ruptura do tronco de uma árvore, pela ação do vento, aumenta com o seu apodrecimento, logo isso pode ter influenciado diretamente no alto número de indivíduos de Espatódea (Spathodea campanulata) danificados no evento meteorológico. Nos indivíduos de Spathodea campanulata arrancados com sistema radicular inteiro pôde ser observado, ainda, baixo volume de sistema radicular (Figura 2), indicando que a árvore possa ter sofrido poda de raízes no passado, além de marcas evidentes desta ocorrência.

O procedimento de poda de raízes é uma das maiores causas de queda de árvores, portanto, qualquer indivíduo que tenha suas raízes podadas para se ajustar a canteiros em calçadas constitui uma árvore de risco. (SEITZ, 2005)

Tibouchina granulosa, por sua vez, apresentou apenas um exemplar com sinais de deterioração por cupins, fator que corrobora para a premissa de que se trata de uma espécie com madeira de baixa resistência mecânica, uma vez que não possui fator atenuante para tamanho dano observado. O mesmo ocorre para a espécie Licania tomentosa, que na literatura é apontada como uma das espécies problemas no meio urbano, devido à quebra de calçadas, entupimento de guias e calhas e conflito com a fiação elétrica (LORENZI, 2002), no entanto, tem sido largamente utilizada, principalmente por suas características favoráveis de copa (TUDINI, 2006). Não há, no entanto, nenhum relato que associe a espécie a rupturas por baixa resistência mecânica, apesar de apresentar valores de densidade básica entre pequeno e médio $\left(0,58 \mathrm{~g} / \mathrm{cm}^{3}\right)$, como obtido por Palharini et al. (2018).

Isso mostra que pode haver influência da limitação de espaço permeável disponível para crescimento vegetativo na queda de árvores, uma vez que espaços exíguos podem aumentar o risco de queda, impedindo o desenvolvimento adequado das estruturas de sustentação da planta (SEITZ, 2005). Condições de plantio sob restrição de espaço estiveram fortemente associadas à queda de árvores em Belo Horizonte, segundo Souza et al. (2019), o que evidencia a dificuldade de ancoragem da planta pelas raízes causada por essa prática.

Alves (2019) em estudo sobre as Espatódeas do campus da Universidade Federal de Viçosa, concluiu que $12 \%$ dos indivíduos sofriam com total restrição de espaço para desenvolvimento de suas raízes, o que pode explicar o grande número de indivíduos dessa espécie danificados na tempestade, bem como grande 
número de indivíduos em más condições fitossanitárias, uma vez que espaço permeável adequado promove a aeração do solo e a disponibilidade de nutrientes para as plantas, condições que contribuem para uma melhor defesa contra patógenos, visto que uma planta saudável está menos susceptível aos mesmos (BIONDI, 1985; SAMPAIO, 2010).

Além disso, indivíduos localizados em ambientes mais pavimentados estão mais expostos às condições desfavoráveis à que as espécies arbóreas têm de tolerar no meio urbano se comparados àqueles plantados em jardins, como à emissão de poluentes pela alta circulação de veículos, compactação do solo, baixo nível de insolação e altas temperaturas, o que reduz seu tempo de vida (OLIVEIRA et al., 2007).

Entende-se, portanto, que a escolha adequada da espécie para determinado espaço é um dos aspectos mais importantes a se considerar no planejamento da arborização em espaços urbanos (SANTOS et al., 2018), considerando que o componente arbóreo deve estar inserido de forma harmônica nos espaços urbanos disponíveis sem que comprometa os demais (SILVA, 2009).

\section{CONCLUSÕES}

A ocorrência de evento climático extremo provocou danos em 145 indivíduos que compõe a arborização do campus da UFV. Tais indivíduos são de 29 espécies e pertencem a 19 famílias botânicas, sendo que 3,3\% das ocorrências foram com palmeiras. As espécies Tibouchina granulosa e Spathodea campanulata representaram mais de $50 \%$ dos indivíduos danificados, ambas as espécies com lenho de baixa resistência mecânica o que pode limitar seu uso para fins de arborização urbana.

Licania tomentosa, por sua vez, contribuiu com $15 \%$ de árvores danificadas no total, apesar de não apresentar relatos desse tipo de problema em outros eventos. Têm-se ainda, que $30 \%$ das árvores danificadas se encontravam acometidas por algum problema fitossanitário que pode ter contribuído para o dano, destas $81 \%$ correspondia a indivíduos de Spathodea campanulata.

Grande parte dos indivíduos danificados (70\%) se localizavam em áreas de calçada, o que permite associar o maior risco de queda às árvores em locais com menor área de canteiro e espaço disponível para o desenvolvimento das raízes. O estudo ressalta a importância da inclusão da arborização urbana no planejamento urbano, a fim de alcançar a compatibilidade entre espécie e ambiente, evitando acidentes diante de eventos imprevisíveis e repensando modelos atuais a fim de buscar o alcance do menor grau de risco associado às árvores urbanas.

\section{REFERÊNCIAS}

ALVES, M. C. A.. Análise de Risco de Spathodea Campanulata no campus Sede da UFV. Monografia (Graduação em Engenharia Florestal) - Universidade Federal de Viçosa, Viçosa, 2019.

BELO HORIZONTE. Subsecretaria de Proteção e Defesa Civil. Belo Horizonte, 2018.

BIONDI, D.. Diagnóstico da arborização de ruas da cidade do Recife. Curitiba: UFPR, 1985.
BRAZOLIN, S.. Biodeterioração, anatomia do lenho e análise de risco de queda de árvores de tipuana, Tipuana tipu (Benth.) O. Kuntze, nos passeios públicos da cidade de São Paulo, SP. Tese (Doutorado) - Escola Superior de Agricultura Luiz de Queiroz, São Paulo, 2009.

CASTRO, P. S.; VALENTE, O. F.; COELHO, D. T.; RAMALHO, R. S.. Interceptação da chuva por mata natural secundária na região de Viçosa, MG. Revista árvore, Viçosa, v.7, n.1, p.7689, 1973. DOI: http://dx.doi.org/10.1590/010067622015000300002 
DUNTEMANN, M.. Elements of an Urban Tree Risk Management Progam. Revista da Sociedade Brasileira de Arborização Urbana, Piracicaba, v.1, n.1, p.99-107, 2006.

EDBERG, R. J.; BERRY, A.. Patterns of structural failures in urban trees: coast live oak (Quercus agrifolia). Journal of Arboriculture, Champaign, v.25, n.1, p.48-55, 1999.

FABIÃO, A. M. D.. A agressividade do meio urbano e algumas medidas de mitigação do stress em árvores das cidades. In WORKSHOP CIRURGIA DAS ÁRVORES. Anais. Lisboa, 1996.

JAMES, K.. Dynamic loading of trees. Journal of Arboriculture, v.29, n.3, p.165-171, 2003.

KLECHOWICZ, N. A.. Diagnóstico dos acidentes com árvores na cidade de Curitiba-PR. Dissertação (Mestrado em Engenharia Florestal) - Universidade Federal do Paraná, Curitiba, 2001.

LOPES, A.; OLIVEIRA, S.; FRAGOSO, M.. Vento Forte e queda de árvores em Lisboa. Avaliação e primeiros resultados, In: ALCOFORADO, M. J.. Estudos sobre Cidades e Alterações Climáticas. CEG, 2008. p.71-91

LORENZI, H.. Árvores Brasileiras: manual de identificação e cultivo de plantas arbóreas do Brasil. 2 ed. Nova Odessa: Instituto Plantarum, 2002.

MOSER, P.; SILVA, A. C.; HIGUCHI, P.; SANTOS, E. M.; SCHMITZ, V.. Avaliação pós-tempestade da Arborização do Campus da Universidade do Estado de Santa Catarina, Lages - SC. Revista da Sociedade Brasileira de Arborização Urbana, n.5, v.22, p.40-51, 2010. DOI: http://dx.doi.org/10.5380/revsbau.v5i2.66270

NILSSON, K.; RANDRUP, T. B.; WANDALL, B. M.. Trees in the urban environment. The forest handbook, Blackwell Science, Oxford, v.1, p.347-361, 2000.

OLIVEIRA, S.; LOPES, A.. Metodologia de avaliação do risco de queda de árvores devido a ventos fortes. O caso de Lisboa. In: CONGRESSO DE GEOGRAFIA PORTUGUESA, 6. Anais. Lisboa, 2007.

PALHARINI, K. M. Z.; GUIMARÃES, J.; FARIA, D. L.; MENDES, R. F.. Potential usage of the urban pruning residue for production of wood based panels. Pesquisas Agrárias e Ambientais, Sinop, v.6, n.3, p.321-325, 2018. DOI: http://dx.doi.org/10.31413/nativa.v6i3.5418

PEREIRA, P.. Estudo de caso do risco de queda de árvores urbanas em via pública na cidade de Dois VizinhosPR. Synergismus Scyentifica, v.6, n.1, p.1-10, 2011.

PORTES, R. C.; FERNANDES FILHO, I. E.; FARIA, A. L. L.; BARBOSA, A. W.. Espacialização das restrições ambientais como subsídio ao plano de desenvolvimento físico e ambiental do campus da Universidade Federal de ViçosaViçosa. In: SIMPÓSIO BRASILEIRO DE GEOGRAFIA FÍSICA APLICADA, 13. Anais. 2009.

RIBEIRO, P.; LOPES, A.. Modelação do risco de queda de árvores sobre as viaturas devido a ventos fortes em Lisboa.
In: CONGRESSO DA GEOGRAFIA PORTUGUESA, 8. Anais.

Lisboa, 2008.

SAMPAIO, A. C. F.; DUARTE, F. G.; SILVA, E. G. C.; ANGELIS, B. L. D.; BLUM, C. T.. Avaliação de árvores de risco na arborização de vias públicas de Nova Olímpia, Paraná. Revista da Sociedade Brasileira de Arborização Urbana, v.5, n.2, p.82-104, 2010. DOI: http://dx.doi.org/10.5380/revsbau.v5i2.66278

SANTOS, F. D.; FANTINEL, R. A.; ZANON, M. L. B.. Arborização Urbana em Frederico Westphalen, RS: Estudo de Caso da Rua do Comércio e Aparício Borges. Revista Gestão \& Sustentabilidade Ambiental, n.7, v.4, p.691-703, 2018. DOI: http://dx.doi.org/10.19177/rgsa.v7e42018691-703

SÃO PAULO. Defesa Civil. São Paulo, 2019.

SEITZ, R.. Avaliação visual de árvores de risco. Curitiba: FUPEF; SBAU, 2005.

SILVA, E. M.. Estudo da arborização urbana do Bairro Mansour, na cidade de Uberlândia/MG. Revista Caminhos de Geografia, v.3, n.5, p.73-83, 2002.

SILVEIRA, L. H. C.; REZENDE, A. V.; VALE, A. T.. Teor de umidade e densidade básica da madeira de nove espécies comerciais amazônicas. Acta Amazônica, Manaus, v.43, n.2, p.179-184, 2013. DOI: http://dx.doi.org/10.1590/S0044$\underline{59672013000200007}$

SOARES JÚNIOR, F. J.. Composição florística e estrutura da vegetação arbórea de um fragmento de Floresta Estacional Semidecidual na Fazenda Tico-Tico, Viçosa-MG. Dissertação (Mestrado em Botânica) - Universidade Federal de Viçosa, Viçosa, 2000.

SOUZA, M. M.; BITTENCOURT, R. A. MARTINI, A. A ocorrência de queda de árvores em Belo Horizonte: análise das condições fitossanitárias dos indivíduos. In: IUFRO, 25. Anais. Curitiba, 2019.

TUDINI, O. G. A arborização de acompanhamento viário e a verticalização na Zona 7 de Maringá - PR. Dissertação (Mestrado em Geografia) - Universidade Estadual de Maringá. Maringá, 2006.

VALE, A. T.; SARMENTO, T. R.; ALMEIDA, A. N.. Caracterização e uso de madeiras de galhos de árvores provenientes da arborização de Brasília, DF. Ciência Florestal, v.15, n.4, p.411-420, 2005. DOI: http://DX.doi.org/10.5902/198050981878

VELOSO, H. P.; RANGEL-FILHO, A. L. R.; LIMA, J. C. A.. Classificação da vegetação brasileira, adaptada a um sistema universal. Rio de Janeiro: IBGE, 1991.

WONG, T. W.; GOOD, J. E. G.; DENNE, M. P.. Tree root damage to pavements and kerbs in the city of Manchester. Arboricultural Journal, v.12, n.1, p.17-34, 1988. DOI: http://doi.org/10.1080/03071375.1988.9756374

YOUNG, C. W. T.. The external signs of decay in trees (Arboricultural Leaflet). London, 1984.

A CBPC - Companhia Brasileira de Produção Científica (CNPJ: 11.221.422/0001-03) detém os direitos materiais desta publicação. Os direitos referem-se à publicação do trabalho em qualquer parte do mundo, incluindo os direitos às renovações, expansões e disseminações da contribuição, bem como outros direitos subsidiários. Todos os trabalhos publicados eletronicamente poderão posteriormente ser publicados em coletâneas impressas sob coordenação da Sustenere Publishing, da Companhia Brasileira de Produção Científica e seus parceiros autorizados. Os (as) autores (as) preservam os direitos autorais, mas não têm permissão para a publicação da contribuição em outro meio, impresso ou digital, em português ou em tradução. 